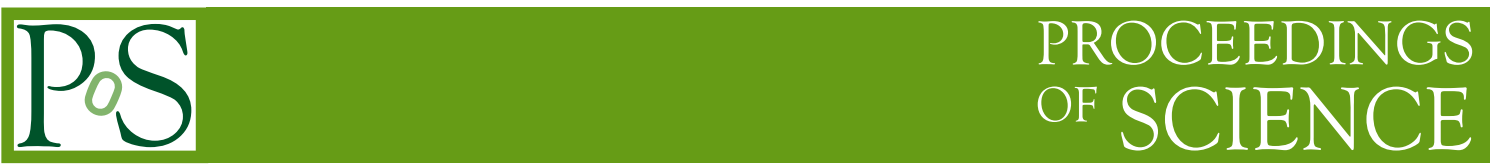

\title{
Bessel Weighted Asymmetries
}

\author{
Harut Avakian \\ Jefferson Lab, 12000 Jefferson Avenue, Newport News, Virginia 23606, USA \\ E-mail: avakian@jlab.org
}

\section{Leonard Gamberg}

Division of Science, Penn State Berks, Reading, PA 19610, USA

E-mail: lpg10epsu.edu

\section{Patrizia Rossi}

INFN, Laboratori Nazionali di Frascati, 00044 Frascati, Italy

Jefferson Lab, 12000 Jefferson Avenue, Newport News, Virginia 23606, USA

E-mail: rossiajlab.org

\section{Alexei Prokudin*}

Division of Science, Penn State Berks, Reading, PA 19610, USA

E-mail: prokudinejlab.org

\begin{abstract}
We review the concept of Bessel weighted asymmetries for semi-inclusive deep inelastic scattering and focus on the cross section in Fourier space, conjugate to the outgoing hadron's transverse momentum, where convolutions of transverse momentum dependent parton distribution functions and fragmentation functions become simple products. Individual asymmetric terms in the cross section can be projected out by means of a generalized set of weights involving Bessel functions. The procedure is applied to studies of the double longitudinal spin asymmetry in semi-inclusive deep inelastic scattering using a new dedicated Monte Carlo generator which includes quark intrinsic transverse momentum within the generalized parton model. We observe a few percent systematic offset of the Bessel-weighted asymmetry obtained from Monte Carlo extraction compared to input model calculations, which is due to the limitations imposed by the energy and momentum conservation at the given energy and hard scale $Q^{2}$. We find that the Bessel weighting technique provides a powerful and reliable tool to study the Fourier transform of TMDs with controlled systematics due to experimental acceptances and resolutions with different TMD model inputs.
\end{abstract}

QCD Evolution 2015 -QCDEV2015-

26-30 May 2015

Jefferson Lab (JLAB), Newport News Virginia, USA

${ }^{*}$ Speaker. 


\section{Introduction}

One of the main goals of hadron physics is to provide a partonic description of the threedimensional (3D) momentum structure of protons and neutrons. The nucleon structure is being studied with many dedicated experiments, recent (HERMES at DESY, Halls A,B and C experiments at Jefferson Lab), running (COMPASS at CERN, STAR and PHENIX at RHIC), approved (JLab $12 \mathrm{GeV}$ upgrade [1], COMPASS-II [2]) or planned (Electron Ion Collider [3, 4, 5]).

The 3D momentum picture of the nucleon is described by the Transverse Momentum Dependent parton distribution functions and fragmentation functions. These Transverse Momentum Dependent distribution and fragmentation functions (collectively here called "TMDs") can be accessed in several types of processes, one of the most important is single particle hadron production in Semi-Inclusive Deep Inelastic Scattering (SIDIS) of leptons on nucleons. A significant amount of data on spin-azimuthal distributions of hadrons in SIDIS, providing access to TMDs has been accumulated in recent years by several collaborations, including HERMES, COMPASS and Halls A,B and C at Jefferson Lab [6, 7, 8, 9, 10, 11, 12, 13, 14, 15]. At least an order of magnitude more data is expected in coming years of running of JLab 12 [1].

QCD factorization theorems generically allow one to separate the short and long distance physics, and thus encode information about internal nucleon structure in individual TMDs [16, $17,18,19]$. In the kinematical domain where transverse momentum of the produced hadron is much smaller than the hard scale $Q$, and on the order of $\Lambda_{Q C D}$, i.e. $\Lambda_{Q C D}^{2} \lesssim P_{h \perp}^{2} \ll Q^{2}$, the SIDIS cross section can be expressed in terms of structure functions which are given by convolutions of TMDs where transverse momentum $\left(k_{\perp}\right)$ dependence of TMDs is integrated over and related to the measured value of $P_{h \perp}$. A reliable method to directly access the $k_{\perp}$ dependence of TMDs is very desirable.

In a paper by Boer, Gamberg, Musch, and Prokudin [20], a new technique has been proposed called Bessel weighting, which relies on a model-independent deconvolution of structure functions in terms of Fourier transforms of TMDs from observed azimuthal moments in SIDIS with polarized and unpolarized targets. A first application of the Bessel weighting Ref. [20] was carried out by Aghasyan, Avakian, De Sanctis, Gamberg, Mirazita, Musch, Prokudin, Rossi in Ref. [21].

In these proceedings we will recapitulate findings of Refs. [20,21].

\section{Bessel Weighting}

The SIDIS cross section can be expressed in a model independent way with leptonic $L_{\mu \nu}$ and hadronic tensors $W^{\mu v}[22,23,24,25,26,27]$,

$$
\frac{d \sigma}{d x d y d \psi d z d \phi_{h} d\left|\boldsymbol{P}_{h \perp}\right|^{2}}=\frac{\alpha^{2}}{x y Q^{2}} \frac{y^{2}}{(1-\varepsilon)}\left(1+\frac{\gamma^{2}}{2 x}\right) L_{\mu v} W^{\mu v}
$$


or in terms of a set of 18 structure functions

$$
\begin{aligned}
\frac{d \sigma}{d x d y d \psi d z d \phi_{h} d\left|\boldsymbol{P}_{h \perp}\right|^{2}}=\frac{\alpha^{2}}{x y Q^{2}} \frac{y^{2}}{2(1-\varepsilon)}\left(1+\frac{\gamma^{2}}{2 x}\right)\left\{F_{U U, T}+\varepsilon F_{U U, L}\right. \\
+\quad \sqrt{2 \varepsilon(1+\varepsilon)} \cos \phi_{h} F_{U U}^{\cos \phi_{h}}+\varepsilon \cos \left(2 \phi_{h}\right) F_{U U}^{\cos 2 \phi_{h}} \\
+\lambda_{e} \sqrt{2 \varepsilon(1-\varepsilon)} \sin \phi_{h} F_{L U}^{\sin \phi_{h}} \\
+S_{\|}\left[\sqrt{2 \varepsilon(1+\varepsilon)} \sin \phi_{h} F_{U L}^{\sin \phi_{h}}+\varepsilon \sin \left(2 \phi_{h}\right) F_{U L}^{\sin 2 \phi_{h}}\right] \\
+S_{\|} \lambda_{e}\left[\sqrt{1-\varepsilon^{2}} F_{L L}+\sqrt{2 \varepsilon(1-\varepsilon)} \cos \phi_{h} F_{L L}^{\cos \phi_{h}}\right] \\
+\left|S_{\perp}\right|\left[\sin \left(\phi_{h}-\phi_{S}\right)\left(F_{U T, T}^{\sin \left(\phi_{h}-\phi_{S}\right)}+\varepsilon F_{U T, L}^{\sin \left(\phi_{h}-\phi_{S}\right)}\right)\right. \\
+\varepsilon \sin \left(\phi_{h}+\phi_{S}\right) F_{U T}^{\sin \left(\phi_{h}+\phi_{S}\right)}+\varepsilon \sin \left(3 \phi_{h}-\phi_{S}\right) F_{U T}^{\sin \left(3 \phi_{h}-\phi_{S}\right)} \\
\left.\left.+\sqrt{2 \varepsilon(1+\varepsilon)} \sin \phi_{S} F_{U T}^{\sin \phi_{S}}+\sqrt{2 \varepsilon(1+\varepsilon)} \sin \left(2 \phi_{h}-\phi_{S}\right) F_{U T}^{\sin \left(2 \phi_{h}-\phi_{S}\right)}\right]\right\} \\
+\left|S_{\perp}\right| \lambda_{e}\left[\sqrt{1-\varepsilon^{2}} \cos \left(\phi_{h}-\phi_{S}\right) F_{L T}^{\cos \left(\phi_{h}-\phi_{S}\right)}+\sqrt{2 \varepsilon(1-\varepsilon)} \cos \phi_{S} F_{L T}^{\cos \phi_{S}}\right. \\
\left.\left.+\sqrt{2 \varepsilon(1-\varepsilon)} \cos \left(2 \phi_{h}-\phi_{S}\right) F_{L T}^{\cos \left(2 \phi_{h}-\phi_{S}\right)}\right]\right\}
\end{aligned}
$$

where the first two subscripts of the structure functions $F_{X Y}$ indicate the polarization of the beam and target, and in certain cases, a third sub-script in $F_{X Y, Z}$ indicates the polarization of the virtual photon. The structure functions depend on the the scaling variables $x, z$, the four momentum $Q^{2}=-q^{2}$, where $q=l-l^{\prime}$ is the momentum of the virtual photon, and $l$ and $l^{\prime}$ are the 4-momenta of the incoming and outgoing leptons, respectively. $P_{h \perp}$ is the transverse momentum component of the produced hadron with respect to the virtual photon direction.

The scaling variables have the standard definitions, $x=Q^{2} / 2(P \cdot q), y=(P \cdot q) /(P \cdot l)$, and $z=\left(P \cdot P_{h}\right) /(P \cdot q)$. Further, in Eq. (2.2) $\alpha$ is the fine structure constant; the angle $\psi$ is the azimuthal angle of $l^{\prime}$ around the lepton beam axis with respect to an arbitrary fixed direction [26], and $\phi_{h}$ is the azimuthal angle between the scattering plane formed by the initial and final momenta of the electron and the production plane formed by the transverse momentum of the observed hadron and the virtual photon, whereas $\phi_{S}$ is the azimuthal angle of the transverse spin in the scattering plane [28]. Finally, $\varepsilon$ is the ratio of longitudinal and transverse photon fluxes [27].

At tree-level (parton-model) of the hard photon-quark scattering process, and to leading order in the $1 / Q$ expansion, the hadronic tensor can be written in factorized form as [24, 29, 27]

$$
2 M W^{\mu v}=\sum_{a} e_{a}^{2} \int d^{2} \boldsymbol{p}_{\perp} d^{2} \boldsymbol{K}_{T} \delta^{(2)}\left(z \boldsymbol{p}_{\perp}+\boldsymbol{K}_{T}-\boldsymbol{P}_{h \perp}\right) \operatorname{Tr}\left\{\Phi\left(x, \boldsymbol{p}_{\perp}\right) \gamma^{\mu} \Delta\left(z, \boldsymbol{K}_{T}\right) \gamma^{v}\right\} .
$$


The quark-quark correlator $[30,17]$ in the above equation is defined as

$$
\Phi_{i j}(p, P, S) \equiv \int \frac{d^{4} b}{(2 \pi)^{4}} e^{i p \cdot b}\left\langle P, S\left|\bar{\psi}_{j}(0) \mathscr{U}\left[\mathscr{C}_{b}\right] \psi_{i}(b)\right| P, S\right\rangle
$$

First we use the representation of the $\delta$-function

$$
\boldsymbol{\delta}^{(2)}\left(z \boldsymbol{p}_{\perp}+\boldsymbol{K}_{T}-\boldsymbol{P}_{h \perp}\right)=\int \frac{d^{2} \boldsymbol{b}_{\mathbf{T}}}{(2 \pi)^{2}} e^{i \boldsymbol{b}_{T}\left(z \boldsymbol{p}_{\perp}+\boldsymbol{K}_{T}-\boldsymbol{P}_{h \perp}\right)}
$$

along with the following definitions,

$$
\begin{aligned}
W^{\mu v}\left(\boldsymbol{P}_{h \perp}\right) & \equiv \int \frac{d^{2} \boldsymbol{b}_{T}}{(2 \pi)^{2}} e^{-i \boldsymbol{b}_{T} \cdot \boldsymbol{P}_{h \perp}} \tilde{W}^{\mu v}\left(\boldsymbol{b}_{T}\right) \\
\tilde{\Phi}_{i j}\left(x, z \boldsymbol{b}_{T}\right) & \equiv \int d^{2} \boldsymbol{p}_{\perp} e^{i z \boldsymbol{b}_{T} \cdot \boldsymbol{p}_{\perp}} \Phi_{i j}\left(x, \boldsymbol{p}_{\perp}\right)=\left.\int \frac{d b^{-}}{(2 \pi)} e^{i x P^{+} b^{-}}\left\langle P, S\left|\bar{\psi}_{j}(0) \mathscr{U}\left[\mathscr{C}_{b}\right] \psi_{i}(b)\right| P, S\right\rangle\right|_{b^{+}=0} \\
\tilde{\Delta}_{i j}\left(z, \boldsymbol{b}_{T}\right) & \equiv \int d^{2} \boldsymbol{K}_{T} e^{i \boldsymbol{b}_{T} \cdot \boldsymbol{K}_{T}} \Delta_{i j}\left(z, \boldsymbol{K}_{T}\right)
\end{aligned}
$$

to re-write the leading term in the hadronic tensor in Fourier space,

$$
2 M \tilde{W}^{\mu v}=\sum_{a} e_{a}^{2} \operatorname{Tr}\left(\tilde{\Phi}\left(x, z \boldsymbol{b}_{T}\right) \gamma^{\mu} \tilde{\Delta}\left(z, \boldsymbol{b}_{T}\right) \gamma^{v}\right)
$$

The advantage of the $\boldsymbol{b}_{T}$ space representation is clear: the hadronic tensor is no longer a convolution of $\boldsymbol{p}_{\perp}$ and $\boldsymbol{K}_{T}$ dependent functions but a simple product of $\boldsymbol{b}_{T}$-dependent functions. This motivates us to re-write the entire cross section in terms of the Fourier transform

$$
\frac{d \sigma}{d x d y d \psi d z_{h} d \phi_{h}\left|\boldsymbol{P}_{h \perp}\right| d\left|\boldsymbol{P}_{h \perp}\right|}=\int \frac{d^{2} \boldsymbol{b}_{T}}{(2 \pi)^{2}} e^{-i \boldsymbol{b}_{T} \cdot \boldsymbol{P}_{h \perp}}\left\{\frac{\alpha^{2}}{x y Q^{2}} \frac{y^{2}}{(1-\varepsilon)}\left(1+\frac{\gamma^{2}}{2 x}\right) L_{\mu \nu} \tilde{W}^{\mu \nu}\right\}
$$

The advantage of this procedure is a possibility to access $\boldsymbol{b}_{T}$-dependent TMDs directly experimentally by projecting with appropriate weights. Indeed, we find that generically the SIDIS 
cross-section takes the following form:

$$
\begin{aligned}
& \frac{d \sigma}{d x d y d \phi_{S} d z_{h} d \phi_{h}\left|\boldsymbol{P}_{h \perp}\right| d\left|\boldsymbol{P}_{h \perp}\right|}=\frac{\alpha^{2}}{x y Q^{2}} \frac{y^{2}}{(1-\varepsilon)}\left(1+\frac{\gamma^{2}}{2 x}\right) \int \frac{d\left|\boldsymbol{b}_{T}\right|}{(2 \pi)}\left|\boldsymbol{b}_{T}\right|\{ \\
& +J_{0}\left(\left|\boldsymbol{b}_{T}\right|\left|\boldsymbol{P}_{h \perp}\right|\right) \mathscr{F}_{U U, T}+\varepsilon J_{0}\left(\left|\boldsymbol{b}_{T}\right|\left|\boldsymbol{P}_{h \perp}\right|\right) \mathscr{F}_{U U, L}+\sqrt{2 \varepsilon(1+\varepsilon)} \cos \phi_{h} J_{1}\left(\left|\boldsymbol{b}_{T}\right|\left|\boldsymbol{P}_{h \perp}\right|\right) \mathscr{F}_{U U}^{\cos \phi_{h}} \\
& +\varepsilon \cos \left(2 \phi_{h}\right) J_{2}\left(\left|\boldsymbol{b}_{T}\right|\left|\boldsymbol{P}_{h \perp}\right|\right) \mathscr{F}_{U U}^{\cos \left(2 \phi_{h}\right)}+\lambda_{e} \sqrt{2 \varepsilon(1-\varepsilon)} \sin \phi_{h} J_{1}\left(\left|\boldsymbol{b}_{T}\right|\left|\boldsymbol{P}_{h \perp}\right|\right) \mathscr{F}_{L U}^{\sin \phi_{h}} \\
& +S_{\|}\left[\sqrt{2 \varepsilon(1+\varepsilon)} \sin \phi_{h} J_{1}\left(\left|\boldsymbol{b}_{T}\right|\left|\boldsymbol{P}_{h \perp}\right|\right) \mathscr{F}_{U L}^{\sin \phi_{h}}+\varepsilon \sin \left(2 \phi_{h}\right) J_{2}\left(\left|\boldsymbol{b}_{T}\right|\left|\boldsymbol{P}_{h \perp}\right|\right) \mathscr{F}_{U L}^{\sin 2 \phi_{h}}\right] \\
& +S_{\|} \lambda_{e}\left[\sqrt{1-\varepsilon^{2}} J_{0}\left(\left|\boldsymbol{b}_{T}\right|\left|\boldsymbol{P}_{h \perp}\right|\right) \mathscr{F}_{L L}+\sqrt{2 \varepsilon(1-\varepsilon)} \cos \phi_{h} J_{1}\left(\left|\boldsymbol{b}_{T}\right|\left|\boldsymbol{P}_{h \perp}\right|\right) \mathscr{F}_{L L}^{\cos \phi_{h}}\right] \\
& +\left|\boldsymbol{S}_{\perp}\right|\left[\sin \left(\phi_{h}-\phi_{S}\right) J_{1}\left(\left|\boldsymbol{b}_{T}\right|\left|\boldsymbol{P}_{h \perp}\right|\right)\left(\mathscr{F}_{U T, T}^{\sin \left(\phi_{h}-\phi_{S}\right)}+\varepsilon \mathscr{F}_{U T, L}^{\sin \left(\phi_{h}-\phi_{S}\right)}\right)\right. \\
& +\varepsilon \sin \left(\phi_{h}+\phi_{S}\right) J_{1}\left(\left|\boldsymbol{b}_{T}\right|\left|\boldsymbol{P}_{h \perp}\right|\right) \mathscr{F}_{U T}^{\sin \left(\phi_{h}+\phi_{S}\right)}+\varepsilon \sin \left(3 \phi_{h}-\phi_{S}\right) J_{3}\left(\left|\boldsymbol{b}_{T}\right|\left|\boldsymbol{P}_{h \perp}\right|\right) \mathscr{F}_{U T}^{\sin \left(3 \phi_{h}-\phi_{S}\right)} \\
& +\sqrt{2 \varepsilon(1+\varepsilon)} \sin \phi_{S} J_{1}\left(\left|\boldsymbol{b}_{T}\right|\left|\boldsymbol{P}_{h \perp}\right|\right) \mathscr{F}_{U T}^{\sin \phi_{S}} \\
& \left.+\sqrt{2 \varepsilon(1+\varepsilon)} \sin \left(2 \phi_{h}-\phi_{S}\right) J_{2}\left(\left|\boldsymbol{b}_{T}\right|\left|\boldsymbol{P}_{h \perp}\right|\right) \mathscr{F}_{U T}^{\sin \left(2 \phi_{h}-\phi_{S}\right)}\right] \\
& +\left|\boldsymbol{S}_{\perp}\right| \lambda_{e}\left[\sqrt{1-\varepsilon^{2}} \cos \left(\phi_{h}-\phi_{S}\right) J_{1}\left(\left|\boldsymbol{b}_{T}\right|\left|\boldsymbol{P}_{h \perp}\right|\right) \mathscr{F}_{L T}^{\cos \left(\phi_{h}-\phi_{S}\right)}\right. \\
& +\sqrt{2 \varepsilon(1-\varepsilon)} \cos \phi_{S} J_{0}\left(\left|\boldsymbol{b}_{T}\right|\left|\boldsymbol{P}_{h \perp}\right|\right) \mathscr{F}_{L T}^{\cos \phi_{S}} \\
& \left.\left.+\sqrt{2 \varepsilon(1-\varepsilon)} \cos \left(2 \phi_{h}-\phi_{S}\right) J_{2}\left(\left|\boldsymbol{b}_{T}\right|\left|\boldsymbol{P}_{h \perp}\right|\right) \mathscr{F}_{L T}^{\cos \left(2 \phi_{h}-\phi_{S}\right)}\right]\right\} \text {. }
\end{aligned}
$$

The structure of the cross section is what one gets from a multipole expansion in $\boldsymbol{b}_{T}$-space followed by a Fourier transform. Each of the structure functions $\mathscr{F}_{X Y, Z}$ in $\boldsymbol{b}_{T}$-space corresponds to the Hankel (or Fourier-Bessel) transform of the corresponding structure function $F_{X Y, Z}^{\ldots}$ in the usual momentum space representation of the cross section. The combinations $\sin \left(n \phi_{h}+\ldots\right) J_{n}\left(\left|\boldsymbol{b}_{T}\right|\left|\boldsymbol{P}_{h \perp}\right|\right)$ and $\cos \left(n \phi_{h}+\ldots\right) J_{n}\left(\left|\boldsymbol{b}_{T}\right|\left|\boldsymbol{P}_{h \perp}\right|\right)$ act as basis functions of the combined transform to $\left(\left|\boldsymbol{P}_{h \perp}\right|, \phi_{h}\right)$ space. Due to the fact that the multipole expansion of the physical cross section terminates, only a finite number of terms appear in the cross section, with $J_{3}$ being the Bessel function of highest order. The structures $\mathscr{F}_{X Y, Z}$ are functions of $\left|\boldsymbol{b}_{T}\right|, x$ and $z$, but no longer depend on the angular variables. Introducing a short-hand notation for products

$$
\mathscr{P}\left[\tilde{f}^{(n)} \tilde{D}^{(m)}\right] \equiv x_{B} \sum_{a} e_{a}^{2}\left(z M\left|\boldsymbol{b}_{T}\right|\right)^{n}\left(z M_{h}\left|\boldsymbol{b}_{T}\right|\right)^{m} \tilde{f}^{a(n)}\left(x, z^{2} \boldsymbol{b}_{T}{ }^{2}\right) \tilde{D}^{a(m)}\left(z, \boldsymbol{b}_{T}{ }^{2}\right)
$$

the leading twist tree level analysis reveals that the Fourier transformed structures in the cross 
section are simple products of TMD PDFs and TMD FFs

$$
\begin{aligned}
\mathscr{F}_{U U, T} & =\mathscr{P}\left[\tilde{f}_{1}^{(0)} \tilde{D}_{1}^{(0)}\right], \\
\mathscr{F}_{U T, T}^{\sin \left(\phi_{h}-\phi_{S}\right)} & =-\mathscr{P}\left[\tilde{f}_{1 T}^{\perp(1)} \tilde{D}_{1}^{(0)}\right], \\
\mathscr{F}_{L L} & =\mathscr{P}\left[\tilde{g}_{1 L}^{(0)} \tilde{D}_{1}^{(0)}\right], \\
\mathscr{F}_{L T}^{\cos \left(\phi_{h}-\phi_{s}\right)} & =\mathscr{P}\left[\tilde{g}_{1 T}^{(1)} \tilde{D}_{1}^{(0)}\right], \\
\mathscr{F}_{U T}^{\sin \left(\phi_{h}+\phi_{S}\right)} & =\mathscr{P}\left[\tilde{h}_{1}^{(0)} \tilde{H}_{1}^{\perp(1)}\right], \\
\mathscr{F}_{U U}^{\cos \left(2 \phi_{h}\right)} & =\mathscr{P}\left[\tilde{h}_{1}^{\perp(1)} \tilde{H}_{1}^{\perp(1)}\right], \\
\mathscr{F}_{U L}^{\sin \left(2 \phi_{h}\right)} & =\mathscr{P}\left[\tilde{h}_{1 L}^{\perp(1)} \tilde{H}_{1}^{\perp(1)}\right], \\
\mathscr{F}_{U T}^{\sin \left(3 \phi_{h}-\phi_{S}\right)} & =\frac{1}{4} \mathscr{P}\left[\tilde{h}_{1 T}^{\perp(2)} \tilde{H}_{1}^{\perp(1)}\right] .
\end{aligned}
$$

Transverse momentum weighted SSA [24, 31, 25] provide a means to disentangle in a model independent way the cross sections and asymmetries in terms of the transverse (momentum) moments of TMD PDFs. Generally they are given by

$$
A_{X Y}^{\mathscr{W}}=2 \frac{\int d\left|\boldsymbol{P}_{h \perp}\right|\left|\boldsymbol{P}_{h \perp}\right| d \phi_{h} d \phi_{S} \mathscr{W}\left(\left|\boldsymbol{P}_{h \perp}\right|, \phi_{h}, \phi_{S}\right)\left(d \sigma^{\uparrow}\left(\phi_{h}, \phi_{S}\right)-d \sigma^{\downarrow}\left(\phi_{h}, \phi_{S}\right)\right)}{\int d\left|\boldsymbol{P}_{h \perp}\right|\left|\boldsymbol{P}_{h \perp}\right| d \phi_{h} d \phi_{S}\left(d \sigma^{\uparrow}\left(\phi_{h}, \phi_{S}\right)+d \sigma^{\downarrow}\left(\phi_{h}, \phi_{S}\right)\right)},
$$

where the labels $X, Y$ represent the polarization, "un" $(U)$, longitudinally $(L)$ and transversely $(T)$ of the beam and target, respectively. The angles $\phi_{S}$ and $\phi_{h}$ specify the directions of the hadron spin polarization and the transverse hadron momentum respectively, relative to the lepton scattering plane. The cross sections $d \sigma^{\uparrow}$ and $d \sigma^{\downarrow}$ correspond to the cases with opposite transverse spin polarization of the target hadron. We have introduced the short-hand notation $\mathscr{W}$ which is a function containing various powers and $\boldsymbol{P}_{h \perp}$ as well as angular dependences of the form $\sin \left(m \phi_{h} \pm n \phi_{S}\right)$ or $\cos \left(m \phi_{h} \pm n \phi_{S}\right)$. For the conventional weighted Sivers asymmetry, $\mathscr{W} \equiv w_{1} \sin \left(\phi_{h}-\phi_{S}\right)$, where $w_{1}=\left|\boldsymbol{P}_{h \perp}\right| / z M$.

Based on the expansion of the SIDIS cross section in terms of Bessel functions $J_{n}$ of transverse momentum and impact parameter in Eq. (2.11), we exploit the orthogonality to generalize the weighting procedure. Now the weighting is of the form

$$
A_{X Y}^{\mathscr{W}}\left(\mathscr{B}_{T}\right)=2 \frac{\int d\left|\boldsymbol{P}_{h \perp}\right|\left|\boldsymbol{P}_{h \perp}\right| d \phi_{h} d \phi_{S} \mathscr{W}\left(\left|\boldsymbol{P}_{h \perp}\right|, \phi_{h}, \phi_{S}, \mathscr{B}_{T}\right)\left(d \sigma^{\uparrow}-d \sigma^{\downarrow}\right)}{\int d\left|\boldsymbol{P}_{h \perp}\right|\left|\boldsymbol{P}_{h \perp}\right| d \phi_{h} d \phi_{S} J_{0}\left(\left|\boldsymbol{P}_{h \perp}\right| \mathscr{B}_{T}\right)\left(d \sigma^{\uparrow}+d \sigma^{\downarrow}\right)}
$$

where the weight function $\mathscr{W}$ corresponds to that of conventional weighted asymmetries, except that we replace

$$
\left|\boldsymbol{P}_{h \perp}\right|^{n} \rightarrow J_{n}\left(\left|\boldsymbol{P}_{h \perp}\right| \mathscr{B}_{T}\right) n !\left(\frac{2}{\mathscr{B}_{T}}\right)^{n} .
$$

Taking the asymptotic form of the Bessel function the conventional weights [31, 25] which are $\propto\left|\boldsymbol{P}_{h \perp}\right|^{n}$ appear as the leading term of the Taylor expansion of the right hand side of Eq. (2.23). Furthermore we note that the parameter $\mathscr{B}_{T}>0$ regularizes UV divergences in moments of TMD PDFs and FFs. More importantly, we will show that the parameter $\mathscr{B}_{T}>0$ allows us to scan TMD PDFs and TMD FFs in Fourier space. In fact, the form of Eq. (2.22) already indicates that 
the weighting implements a Fourier-decomposition of the cross section in transverse momentum space.

We will illustrate this method for the Sivers Bessel weighted asymmetry. One can see from Eq. (2.11) that the appropriate weight for the Sivers asymmetry is

$$
\mathscr{W}=\frac{2 J_{1}\left(\left|\boldsymbol{P}_{h \perp}\right| \mathscr{B}_{T}\right)}{z M \mathscr{B}_{T}} \sin \left(\phi_{h}-\phi_{S}\right), \quad \text { i.e., } \quad w_{1}=\frac{2 J_{1}\left(\left|\boldsymbol{P}_{h \perp}\right| \mathscr{B}_{T}\right)}{z M \mathscr{B}_{T}},
$$

corresponding to $\left|\boldsymbol{P}_{h \perp}\right| / z M$ in the limit $\left|\boldsymbol{P}_{h \perp}\right| \ll 1 / \mathscr{B}_{T}$. Then the Bessel-weighted Sivers asymmetry is

$$
A_{U T}^{\frac{2 J_{1}\left(\left|\boldsymbol{P}_{h \perp}\right| \mathscr{B}_{T}\right)}{{ }^{M \mathscr{B}_{T}}} \sin \left(\phi_{h}-\phi_{S}\right)}\left(\mathscr{B}_{T}\right)=2 \frac{\int d\left|\boldsymbol{P}_{h \perp}\right|\left|\boldsymbol{P}_{h \perp}\right| d \phi_{h} d \phi_{S} \frac{2 J_{1}\left(\left|\boldsymbol{P}_{h \perp}\right| \mathscr{B}_{T}\right)}{z M \mathscr{B}_{T}} \sin \left(\phi_{h}-\phi_{S}\right)\left(d \sigma^{\uparrow}-d \sigma^{\downarrow}\right)}{\int d\left|\boldsymbol{P}_{h \perp}\right|\left|\boldsymbol{P}_{h \perp}\right| d \phi_{h} d \phi_{S} J_{0}\left(\left|\boldsymbol{P}_{h \perp}\right| \mathscr{B}_{T}\right)\left(d \sigma^{\uparrow}+d \sigma^{\downarrow}\right)}
$$

where the axially symmetric denominator is given by

$$
\int d\left|\boldsymbol{P}_{h \perp}\right|\left|\boldsymbol{P}_{h \perp}\right| d \phi_{h} d \phi_{S} J_{0}\left(\left|\boldsymbol{P}_{h \perp}\right| \mathscr{B}_{T}\right) \int \frac{d\left|\boldsymbol{b}_{T}\right|}{(2 \pi)}\left|\boldsymbol{b}_{T}\right| J_{0}\left(\left|\boldsymbol{b}_{T}\right|\left|\boldsymbol{P}_{h \perp}\right|\right) \mathscr{F}_{U U, T}
$$

and from Eq. (2.11) the numerator is

$$
\int d\left|\boldsymbol{P}_{h \perp}\right|\left|\boldsymbol{P}_{h \perp}\right| d \phi_{h} d \phi_{S} \frac{2 J_{1}\left(\left|\boldsymbol{P}_{h \perp}\right| \mathscr{B}_{T}\right)}{z M \mathscr{B}_{T}} \sin ^{2}\left(\phi_{h}-\phi_{s}\right) \int \frac{d\left|\boldsymbol{b}_{T}\right|}{(2 \pi)}\left|\boldsymbol{b}_{T}\right| J_{1}\left(\left|\boldsymbol{b}_{T}\right|\left|\boldsymbol{P}_{h \perp}\right|\right) \mathscr{F}_{U T}^{\sin \left(\phi_{h}-\phi_{s}\right)} .
$$

Finally, making use of the closure relation of the Bessel function we obtain $\mathrm{f}$ the Bessel weighted Sivers asymmetry,

$$
A_{U T, T}^{\frac{2 J_{1}\left(\left|\boldsymbol{P}_{h \perp}\right| \mathscr{B}_{T}\right)}{{ }^{M \mathscr{S}_{T}}} \sin \left(\phi_{h}-\phi_{s}\right)}\left(\mathscr{B}_{T}\right)=-2 \frac{\sum_{a} e_{a}^{2} H_{U T, T}^{\sin \left(\phi_{h}-\phi_{S}\right)}\left(Q^{2}, \mu^{2}\right) \tilde{f}_{1 T}^{\perp(1) a}\left(x, z^{2} \mathscr{B}_{T}^{2} ; \mu^{2}, \zeta\right) \tilde{D}_{1}^{(0) a}\left(z, \mathscr{B}_{T}^{2} ; \mu^{2}, \hat{\zeta}\right)}{\sum_{a} e_{a}^{2} H_{U U, T}\left(Q^{2}, \mu^{2}\right) \tilde{f}_{1}^{(0) a}\left(x, z^{2} \mathscr{B}_{T}^{2} ; \mu^{2}, \zeta\right) \tilde{D}_{1}^{(0) a}\left(z, \mathscr{B}_{T}^{2} ; \mu^{2}, \hat{\zeta}\right)} .
$$

One can see from Eq. 2.28 that the weighed asymmetry is related to a product of Fourier transformed Sivers function and unpolarised TMD fragmentation function.

Details of the method can be found in Ref [20].

\section{Feasibility studies}

We studied experimental feasibility of the method in Ref. [21]. Consider the unpolarized and double longitudinal structure functions,

$$
\mathscr{F}_{U U, T}=x \sum_{a} e_{a}^{2} \tilde{f}_{1}^{a}\left(x, z^{2} \boldsymbol{b}_{T}{ }^{2}\right) \tilde{D}_{1}^{a}\left(z, \boldsymbol{b}_{T}{ }^{2}\right), \mathscr{F}_{L L}=x \sum_{a} e_{a}^{2} \tilde{g}_{1 L}^{a}\left(x, z^{2} \boldsymbol{b}_{T}{ }^{2}\right) \tilde{D}_{1}^{a}\left(z, \boldsymbol{b}_{T}{ }^{2}\right),
$$

where the Fourier transform of the TMDs are defined as

$$
\begin{aligned}
\tilde{f}\left(x, \boldsymbol{b}_{T}^{2}\right) & =\int d^{2} \boldsymbol{k}_{\perp} e^{i \boldsymbol{b}_{T} \cdot \boldsymbol{k}_{\perp}} f\left(x, \boldsymbol{k}_{\perp}^{2}\right) \\
& =2 \pi \int d \boldsymbol{k}_{\perp} \boldsymbol{k}_{\perp} J_{0}\left(\left|\boldsymbol{b}_{T}\right|\left|\boldsymbol{k}_{\perp}\right|\right) f\left(x, \boldsymbol{k}_{\perp}^{2}\right)
\end{aligned}
$$




$$
\begin{aligned}
\tilde{D}\left(z, \boldsymbol{b}_{T}^{2}\right) & =\int d^{2} \boldsymbol{p}_{\perp} e^{i \boldsymbol{b}_{T} \cdot \boldsymbol{p}_{\perp}} D\left(z, \boldsymbol{p}_{\perp}^{2}\right) \\
& =2 \pi \int d \boldsymbol{p}_{\perp} \boldsymbol{p}_{\perp} J_{0}\left(\left|\boldsymbol{b}_{T}\right|\left|\boldsymbol{p}_{\perp}\right|\right) D\left(x, \boldsymbol{p}_{\perp}^{2}\right) .
\end{aligned}
$$

Now we form the double longitudinal spin asymmetry

$$
A_{L L}^{J_{0}\left(b_{T} P_{h \perp}\right)}\left(b_{T}\right)=\frac{\tilde{\sigma}^{+}\left(b_{T}\right)-\tilde{\sigma}^{-}\left(b_{T}\right)}{\tilde{\sigma}^{+}\left(b_{T}\right)+\tilde{\sigma}^{-}\left(b_{T}\right)} \equiv \frac{\tilde{\sigma}_{L L}\left(b_{T}\right)}{\tilde{\sigma}_{U U}\left(b_{T}\right)}=\sqrt{1-\varepsilon^{2}} \frac{\sum_{a} e_{a}^{2} \tilde{g}_{1 L}^{a}\left(x, z^{2} b_{T}^{2}\right) \tilde{D}_{1}^{a}\left(z, b_{T}^{2}\right)}{\sum_{a} e_{a}^{2} \tilde{f}_{1}^{a}\left(x, z^{2} b_{T}^{2}\right) \tilde{D}_{1}^{a}\left(z, b_{T}^{2}\right)},
$$

Note that in our definition $b_{T}$ is Fourier conjugate variable to $P_{h \perp}$ [20].

A Monte Carlo generator is a crucial component in testing experimental procedures such as those described in Eq. (3.4). In order to check the Bessel weighting technique we need a Monte Carlo that generates events in phase space with different TMD model input, which includes explicit dependence on intrinsic parton transverse momentum $k_{\perp}$ and $p_{\perp}$.

In the Monte Carlo generator software, we used the general-purpose, self-adapting event generator, Foam [32], for drawing random points according to an arbitrary, user-defined distribution in $n$-dimensional space.

Implementing the Monte Carlo we generate kinematical distributions in $x, z, k_{\perp}$, and $p_{\perp}$ of SIDIS events for several model inputs of TMDs. These distributions are then used to check the consistency of dependence of extracted quantities under different model assumptions, including, for example Gaussian and non-Gaussian distributions in transverse momentum.

In case the dependence is assumed to be a Gaussian, $x$ and $z$ dependent widths are assumed, so that TMDs take the following form,

$$
\begin{aligned}
& f_{1}\left(x, \boldsymbol{k}_{\perp}^{2}\right)=f_{1}(x) \frac{1}{\left\langle k_{\perp}^{2}(x)\right\rangle_{f_{1}}} \exp \left(-\frac{\boldsymbol{k}_{\perp}^{2}}{\left\langle k_{\perp}^{2}(x)\right\rangle_{f_{1}}}\right), \\
& g_{1 L}\left(x, \boldsymbol{k}_{\perp}^{2}\right)=g_{1 L}(x) \frac{1}{\left\langle k_{\perp}^{2}(x)\right\rangle_{g_{1}}} \exp \left(-\frac{\boldsymbol{k}_{\perp}^{2}}{\left\langle k_{\perp}^{2}(x)\right\rangle_{g_{1}}}\right), \\
& D_{1}\left(z, \boldsymbol{p}_{\perp}^{2}\right)=D_{1}(z) \frac{1}{\left\langle p_{\perp}^{2}(z)\right\rangle} \exp \left(-\frac{\boldsymbol{p}_{\perp}^{2}}{\left\langle p_{\perp}^{2}(z)\right\rangle}\right)
\end{aligned}
$$

where $f(x)$ and $D(z)$ are corresponding collinear parton distribution and fragmentation functions and the widths are $x$ and $z$ dependent functions. In our studies we adopt the modified Gaussian distribution functions and fragmentation functions from Eq. (3.5)-(3.7), in which $x$ and $k_{\perp}$ dependencies are inspired by AdS/QCD results [33, 34], with $\left\langle k_{\perp}^{2}(x)\right\rangle=C x(1-x)$ and $\left\langle p_{\perp}^{2}(z)\right\rangle=D z(1-z)$, where the constants $C$ and $D$ may be different for different flavors and polarization states (see for example [35]). Similarly such non-factorized $x, k_{\perp}$ distribution functions are also suggested by the diquark spectator model [36] and the NJL-jet model [37].

For the $x$ and $z$ dependence in Eqs. (3.5) and (3.7) we use the parametrizations, $f_{1}(x)=(1-$ $x)^{3} x^{-1.313}, g_{1 L}(x)=f_{1}(x) x^{0.7}$, and $D_{1}(z)=0.8(1-z)^{2}$, using input values $C=0.54 \mathrm{GeV}^{2}$ and $D=0.5 \mathrm{GeV}^{2}$. We also assume that $\left\langle k_{\perp}^{2}\right\rangle_{g_{1 L}}=0.8\left\langle k_{\perp}^{2}\right\rangle_{f_{1}}$; this assumption is consistent with lattice studies [38] and experimental measurements [14]. 
As an example of a non-Gaussian $k_{\perp}$ distribution we implement the following one inspired by the shape of the resulting distribution in the light-cone quark model $[39,40]$

$$
f_{1}\left(x, \boldsymbol{k}_{\perp}^{2}\right)=f_{1}(x) /\left(1+20.82 k_{\perp}^{2}+126.7 k_{\perp}^{4}+1285 k_{\perp}^{6}\right) .
$$

where the coefficients for $g_{1 L}\left(x, \boldsymbol{k}_{\perp}^{2}\right)$ are chosen in such a way that effectively $\left\langle k_{\perp}^{2}\right\rangle_{g_{1 L}} /\left\langle k_{\perp}^{2}\right\rangle_{f_{1}}=0.8$.

We then generate events using the cross section for both Gaussian and non-Gaussian initial distributions respectively. Note that the generator we construct is implemented with on mass-shell partons and with four momentum conservation imposed. While this choice is not compulsory we adopt it as it allows us to fully reconstruct kinematics for a given event. At the same time limitations due to available phase space integration will modify the reconstructed distributions with respect to the input distributions. We analyze the effect of the available phase space in the Monte Carlo on the average $\left\langle k_{\perp}^{2}\right\rangle$ for finite beam energies as a function of $x$ by calculating the effective $\left\langle k_{\perp}^{2}\right\rangle$ from the following formula,

$$
\left\langle k_{\perp}^{2}(x)\right\rangle=\frac{\int d^{2} \boldsymbol{k}_{\perp} k_{\perp}^{2} d \sigma_{M C}}{\int d^{2} \boldsymbol{k}_{\perp} d \sigma_{M C}}=\frac{\sum_{j=1}^{N} k_{\perp j}^{2}}{N},
$$

where the index $j$ runs over the $N$ Monte Carlo generated events. Note, $d \sigma_{M C}$ is the cross section of the Monte Carlo simulation modified by imposing the four momenta conservation and on-shell condition for initial quark.

The Monte Carlo generated events are used like experimental events to extract both the Bessel weighted asymmetry, $A_{L L}^{J_{0}\left(b_{T} P_{h \perp}\right)}$, and the ratio of the Fourier transform of $g_{1 L}$ and $f_{1}$ using the Bessel weighting method described in [20]. The results are then compared to the Monte Carlo input. The Bessel moments are extracted from the Monte Carlo with $6 \mathrm{GeV}$ beam energy using both the modified Gaussian type of functions (see Eqs. (3.5)-(3.7)) and power law-tail like function (see Eq. (3.8)).

The numerical results of our studies are summarized and displayed in Figs. 1 and 2 for the modified Gaussian distribution function and for the power law-tail like distribution function inputs respectively. In the left panel of Fig. 1 we show the Bessel-weighted asymmetry versus $b_{T}$. The blue curve labeled "BW Input", is the asymmetry calculated analytically using the right hand side of Eq. (3.4) and the Fourier transformed input distribution functions.

We now compare various distributions generated from the Monte Carlo. We plot the generated distribution (full red points) labeled "BW $\left(P_{h \perp}\right)$ Generated", and the black triangles labeled "BW $\left(P_{h \perp}\right) \mathrm{Sm}+$ Acc", which represents the same extraction after experimental smearing and acceptance (using the CLAS detector [41], which is a quasi- $4 \pi$ detector with less than $1 \%$ momentum resolution in the presented bin $\langle x\rangle=0.22$, and $\langle z\rangle=0.51$ ).

Analyzing our MC results with four momenta conservation and target mass correction, we are able to distinguish two effects in the left panels of Figs. 1 and 2:

1. Solid (blue) curve versus triangular (green) data points: The distributions realized in the MC simulation differ from the input distributions. In the MC, the four-momentum conservation does not allow the variables $\boldsymbol{k}_{\perp}$ and $\boldsymbol{p}_{\perp}$ to be sampled independently over the whole integration range, as it would have to be done to reproduce the unmodified generalized parton model. The actual $\boldsymbol{k}_{\perp}$ and $\boldsymbol{p}_{\perp}$ distributions realized by the MC differ from the analytic 
input distributions Eqns. (3.5)-(3.8) noticeably, especially in their widths. The solid (blue) curve in the left panel of Figs. 1 and 2 is calculated from the input distributions according to the generalized parton model; the FFs on the right hand side of Eq. (3.4) cancel exactly in the single flavor scenario. Thus the solid curve can be compared to the triangle shaped (green) data points, which have also been calculated from a ratio of TMD PDFs, Eq. (??), albeit with the actual distributions realized in the MC.

2. Triangular (green) data points vs. circular (red) data points: inadequacy of the generalized parton model to describe the data. In a single flavor scenario, the distribution functions $\tilde{D}_{1}^{a}$ cancel exactly on the right hand side of Eq. (3.4). Therefore, there should not be any difference between the full asymmetry $A_{L L}^{J_{0}\left(b_{T} P_{h \perp}\right)}\left(b_{T}\right)$ of Eq. (3.4) and the ratio of TMD PDFs. However, we do observe a difference between the circular (red) data points and the triangular (green) data points in the left panels of Figs. 1 and 2. Again, the four-momentum conservation we have implemented is the reason for the observed difference. Since $\boldsymbol{k}_{\perp}$ and $\boldsymbol{p}_{\perp}$ are no longer sampled and the right hand side of Eq. (3.4) looses the prerequisites for its derivation and is violated to some degree. Therefore, we see only an incomplete cancellation of FFs for the Monte Carlo events.

To an experimentalist who is concerned about systematic errors attributed to the observables he or she extracts, the first of the two effects above is not an issue. The purpose of the generalized parton model is to provide a parametrization of the data one observes. Any effect of the underlying
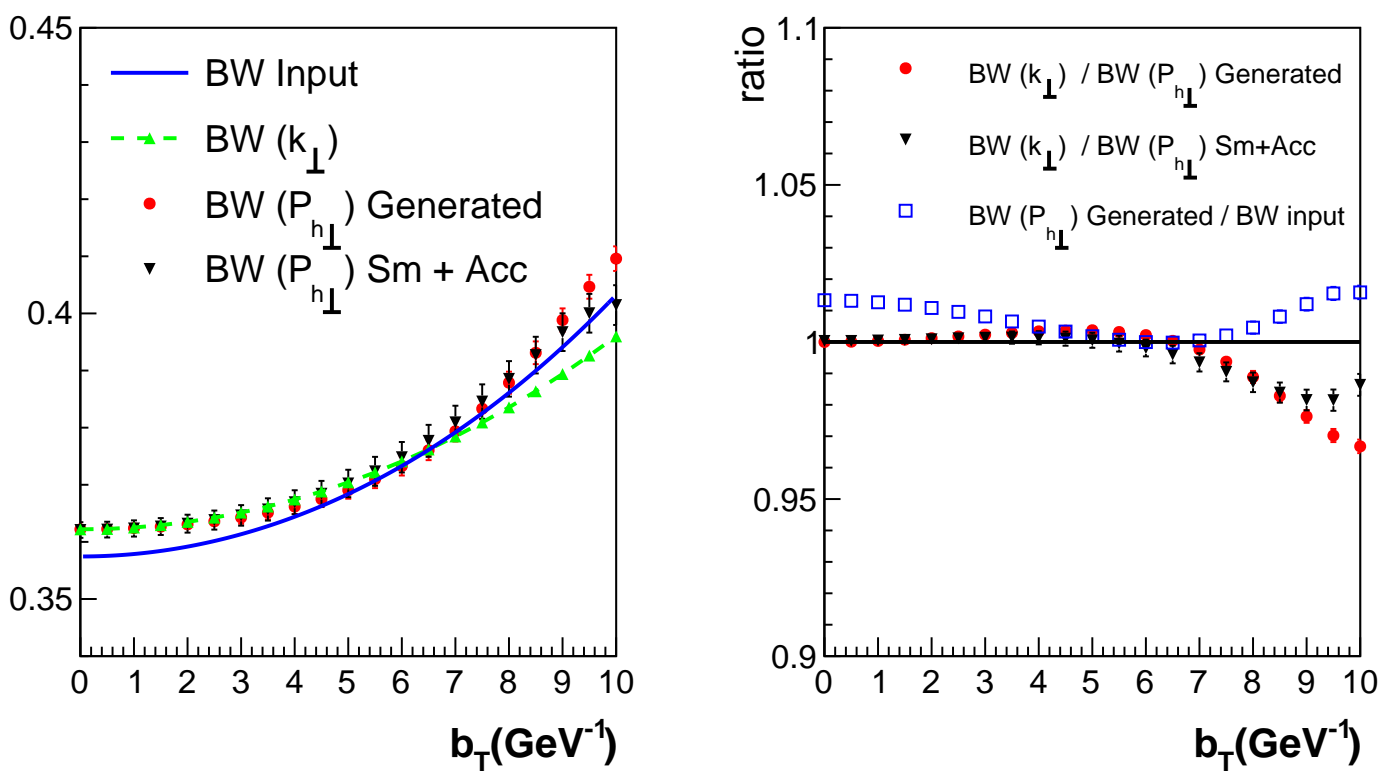

Figure 1: Left panel:The ratio of Fourier transforms $\tilde{g}_{1 L} / \tilde{f}_{1}$ and the Bessel weighted asymmetry $A_{L L}^{J_{0}\left(b_{T} P_{h \perp}\right)}$ plotted versus $b_{T}$. The solid curve (blue) is the Fourier transform of the input to the Monte Carlo given by Eq. (3.4), red points are generated Monte Carlo events, and triangles down (black) represent results of Monte Carlo events after experimental smearing and acceptance at $\langle x\rangle=0.22$, and $\langle z\rangle=0.51$. The triangles up with dashed curve (green) are results of the Monte Carlo without inclusion of fragmentation functions. Right panel: Ratios that represent accuracies of our results. 

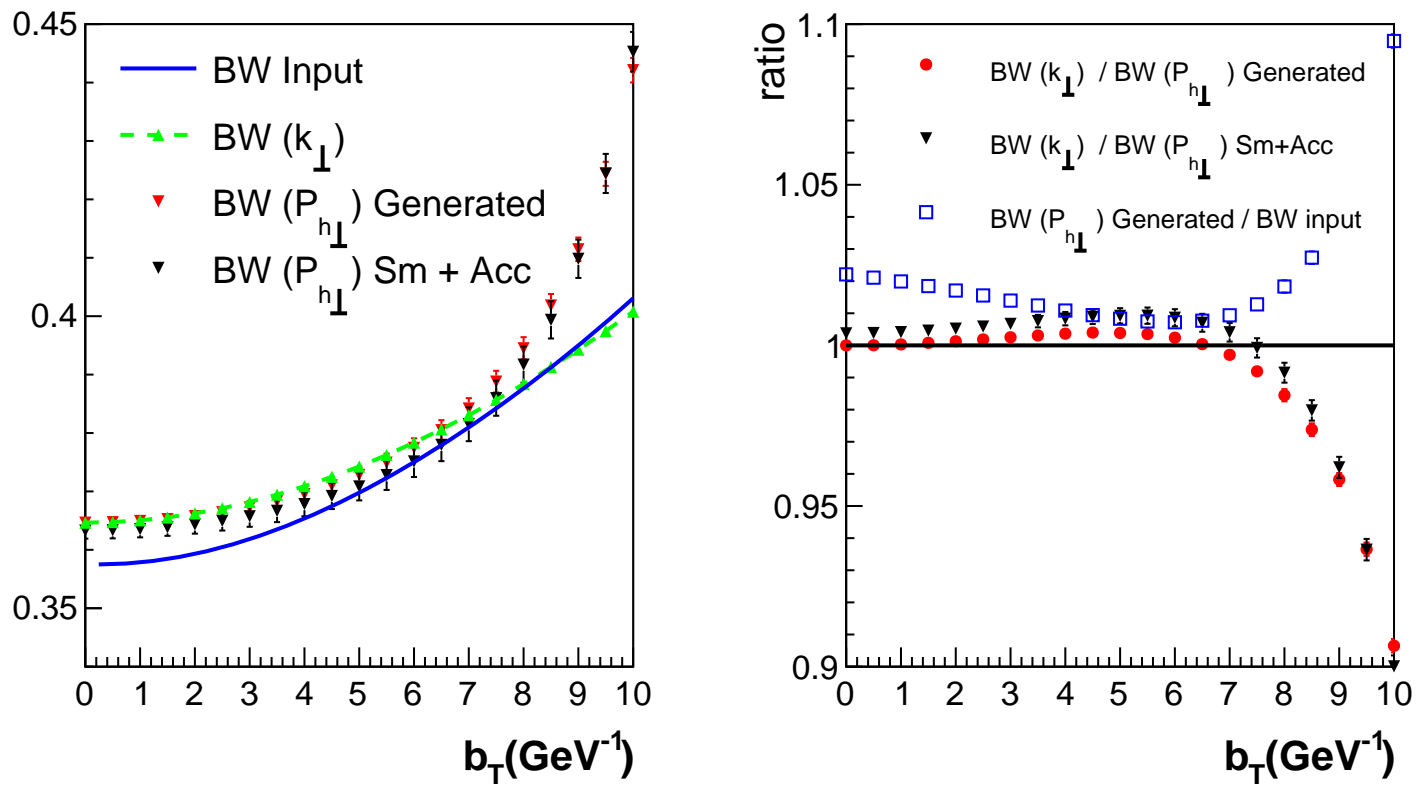

Figure 2: The same as in Fig. (1) but here from the power-law tail distribution function based on the Monte Carlo.

scattering mechanism that can be absorbed into the distributions does not contradict the validity of the model. The only concern one might have is that the distributions become beam energy $/ Q^{2}$ dependent, an issue that should be addressed using TMD evolution equations.

On the other hand, the second effect presented above can be taken as an indication for systematic uncertainties. If, indeed, the physical reality does not generate events in accordance with the functional shape of the generalized parton model, then using the model for the extraction of distributions necessarily involves systematic errors. Again, we point out that it is unclear whether the modifications we have implemented in our MC bring us closer to the physical reality. Nonetheless, the modifications are reasonable and so we believe they can give us a hint about the order of magnitude of systematic errors from the corresponding approximations in the model. One can then estimate that for calculations such as those performed in Ref. [42], systematic errors in the comparison with experimental data for $b_{T}<6 \mathrm{GeV}^{-1}$ are of the order of a few percent. For the data with $b_{T}>6 \mathrm{GeV}^{-1}$, the effects of four-momentum conservation (difference between red and green points) becomes more pronounced, and a fit of data using the generalized parton model without manifest four-momentum conservation therefore becomes less accurate.

\section{Conclusions}

We find that the Bessel weighting technique provides a powerful and reliable tool to study the Fourier transform of TMDs with controlled systematics due to experimental acceptances and resolutions with different TMD models inputs. We plan to expand our studies with more advanced parton shower and fragmentation mechanisms, as well as to include nuclear modifications in our Monte Carlo and extraction procedure. 


\section{References}

[1] J. Dudek et al., Eur.Phys.J. A48, 187 (2012), arXiv:1208.1244.

[2] COMPASS, F. Gautheron et al., (2010).

[3] H. Gao et al., Eur.Phys.J.Plus 126, 2 (2011), arXiv:1009.3803.

[4] D. Boer et al., (2011), arXiv:1108.1713.

[5] A. Accardi et al., (2012), arXiv:1212.1701.

[6] HERMES, A. Airapetian et al., Phys. Rev. Lett. 94, 012002 (2005), hep-ex/0408013.

[7] COMPASS, V. Y. Alexakhin et al., Phys. Rev. Lett. 94, 202002 (2005), hep-ex/0503002.

[8] COMPASS, E. S. Ageev et al., Nucl. Phys. B765, 31 (2007), hep-ex/0610068.

[9] HERMES, A. Airapetian et al., Phys. Lett. B693, 11 (2010), arXiv:1006.4221.

[10] The COMPASS, M. G. Alekseev et al., Phys. Lett. B692, 240 (2010), arXiv:1005.5609.

[11] M. G. Alekseev et al., Eur. Phys. J. C70, 39 (2010), arXiv:1007.1562.

[12] COMPASS Collaboration, C. Adolph et al., Phys.Lett. B713, 10 (2012), arXiv:1202.6150.

[13] H. Mkrtchyan et al., Phys. Lett. B665, 20 (2008), arXiv:hep-ph/0709.3020.

[14] CLAS, H. Avakian et al., Phys. Rev. Lett. 105, 262002 (2010), arXiv:hep-ex/1003.4549.

[15] The Jefferson Lab Hall A Collaboration, X. Qian et al., Phys.Rev.Lett. 107, 072003 (2011), arXiv:1106.0363, 6 pages, 2 figures, 2 tables, published in PRL.

[16] J. C. Collins and D. E. Soper, Nucl. Phys. B193, 381 (1981).

[17] J. C. Collins and D. E. Soper, Nucl.Phys. B194, 445 (1982).

[18] J. C. Collins, D. E. Soper, and G. F. Sterman, Nucl.Phys. B250, 199 (1985).

[19] J. Collins, (2011), Foundations of Perturbative QCD, Cambridge, UK: Univ.Pr.

[20] D. Boer, L. Gamberg, B. Musch, and A. Prokudin, JHEP 1110, 021 (2011), arXiv:1107.5294.

[21] M. Aghasyan et al., JHEP 03, 039 (2015), arXiv:1409.0487.

[22] M. Gourdin, Nucl. Phys. B49, 501 (1972).

[23] A. Kotzinian, Nucl. Phys. B441, 234 (1995), hep-ph/9412283.

[24] P. J. Mulders and R. D. Tangerman, Nucl. Phys. B461, 197 (1996), hep-ph/9510301.

[25] D. Boer and P. J. Mulders, Phys. Rev. D57, 5780 (1998), hep-ph/9711485.

[26] M. Diehl and S. Sapeta, Eur. Phys. J. C41, 515 (2005), arXiv:hep-ph/0503023.

[27] A. Bacchetta et al., JHEP 02, 093 (2007), arXiv:hep-ph/0611265.

[28] A. Bacchetta, U. D’Alesio, M. Diehl, and C. A. Miller, Phys. Rev. D70, 117504 (2004), arXiv:hep-ph/0410050.

[29] D. Boer, P. J. Mulders, and F. Pijlman, Nucl. Phys. B667, 201 (2003), hep-ph/0303034.

[30] D. E. Soper, Phys. Rev. Lett. 43, 1847 (1979).

[31] A. Kotzinian and P. Mulders, Phys.Lett. B406, 373 (1997), arXiv:hep-ph/9701330. 
[32] S. Jadach, Computer Physics Communications 152, 55 (2003), arXiv:physics/0203033.

[33] S. J. Brodsky, U of Warsaw, July 3-6 (2012).

[34] G. F. de Teramond and S. J. Brodsky, Phys.Rev.Lett. 102, 081601 (2009), arXiv:0809.4899.

[35] A. Signori, A. Bacchetta, M. Radici, and G. Schnell, JHEP 1311, 194 (2013), arXiv:1309.3507.

[36] L. P. Gamberg, G. R. Goldstein, and M. Schlegel, Phys. Rev. D77, 094016 (2008), arXiv:hep-ph/0708.0324.

[37] H. H. Matevosyan, W. Bentz, I. C. Cloet, and A. W. Thomas, Phys.Rev. D85, 014021 (2012), arXiv:1111.1740.

[38] B. U. Musch, P. Hagler, J. W. Negele, and A. Schafer, Phys.Rev. D83, 094507 (2011), arXiv:1011.1213.

[39] B. Pasquini and S. Boffi, Phys.Rev. D76, 074011 (2007), arXiv:0707.2897.

[40] B. Pasquini, S. Boffi, A. Efremov, and P. Schweitzer, (2009), arXiv:0912.1761.

[41] CLAS Collaboration, B. Mecking et al., Nucl.Instrum.Meth. A503, 513 (2003).

[42] Z. Lu and B.-Q. Ma, Phys.Rev. D87, 034037 (2013), arXiv:1212.6864. 